\title{
Artículo
}

\section{Evaluación del sistema archivístico del Hospital de León}

\author{
Por Ma del Carmen Rodríguez López, Ángela Díez Díez, Josefa Gallego Lorenzo, Marian Morán Suárez
} y Lourdes Santos de Paz

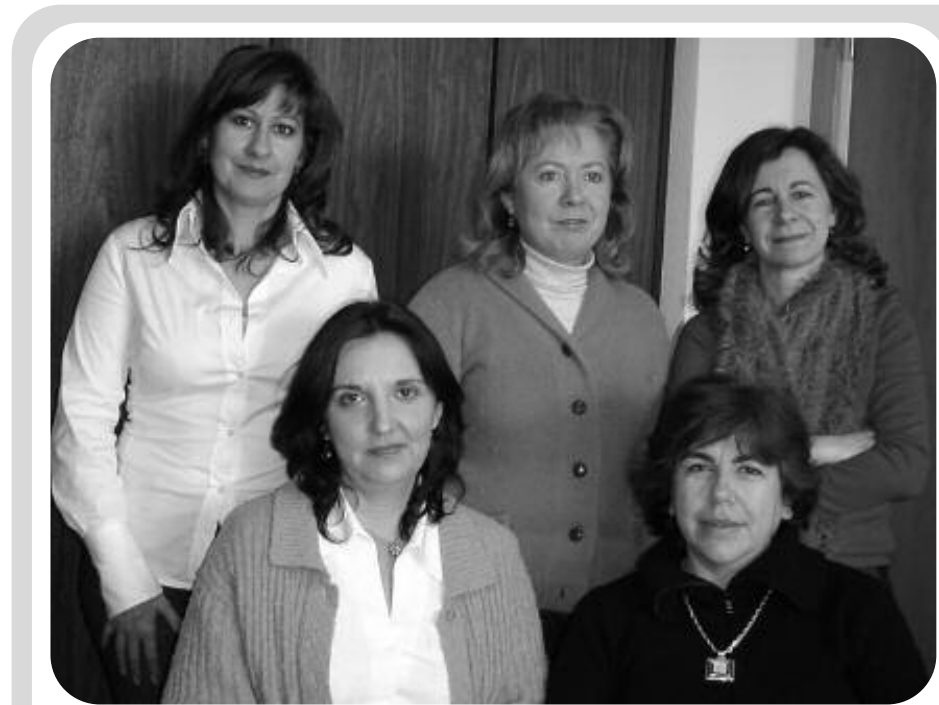

María del Carmen Rodríguez López, licenciada en filosofía y letras (sección historia). Doctora en historia y profesora titular de la Diplomatura de biblioteconomía y documentación en la Universidad de León (ULE). Sus últimos proyectos de investigación y publicaciones han tenido por objeto los sistemas archivísticos, la ponderación del valor económico generado por la gestión de los archivos, así como la implantación del grado en documentación en la ULE.

Ángela Díez Díez, profesora titular de escuela universitaria, Departamento de Ingeniería Eléctrica y Electrónica. Ingeniera técnica industrial por la Universidad de León (ULE). Master en sistemas informáticos por la Universidad Pontificia de Madrid. Licenciada en informática por la ULE. Imparte clase desde 1991 en la Diplomatura de biblioteconomía y documentación y en Industriales e informática de la ULE. Participa de forma activa en diversos proyectos de investigación sobre empleo de nuevas tecnologías y en el tratamiento y la adquisición de información y de bases de datos. Imparte cursos dirigidos a empresas.

Josefa Gallego Lorenzo, licenciada en geografía e historia (sección arte) por la Universidad de Santiago de Compostela y doctora en historia del arte por la Universidad de León (ULE); master en documentación digital por la Universidad Pompeu Fabra. Profesora asociada del área de biblioteconomía y documentación de la ULE. Sus líneas de investigación se hallan relacionadas con la bibliografía, las fuentes y recursos de información en humanidades, habiendo publicado varios trabajos en congresos y revistas.

Marian Morán Suárez, licenciada en sociología y doctora por la Universidad de León (ULE). Ha trabajado como documentalista, y actualmente es profesora del área de biblioteconomía y documentación de la ULE. Entre sus líneas de investigación figuran: necesidades de información y producción científica, y organización del conocimiento y su representación.

Lourdes Santos de Paz es licenciada en filosofía y letras (sección filología) y diplomada en biblioteconomía y documentación por la Universidad de León (ULE). Profesora titular de la Diplomatura de biblioteconomía y documentación en la ULE. Las líneas de investigación en las que ha trabajado son: sistemas públicos de bibliotecas, conservación documental, habiendo publicado diversos trabajos sobre estos temas. Participa en proyectos de desarrollo, uso y calidad de publicaciones electrónicas.
Resumen: Investigación sobre los orígenes y evolución de los tres centros hospitalarios que forman el Hospital de León, reunidos en el Sistema Asistencial de Castilla y León. El conocimiento de la procedencia es fundamental para la correcta organización de los archivos de historias clínicas y los documentos que las integran. Se estudió además la gestión de los archivos de historias clínicas del Hospital de León para determinar la importancia que tiene la adecuada localización conceptual y física de las mismas en el desenvolvimiento diario de los centros hospitalarios. De esta manera es posible estimar la cuantía económica que supone la correcta gestión del archivo para evitar la prolongación de la estancia del paciente y la necesidad de repetir las pruebas.

Palabras clave: Sistema archivístico sanitario, Gestión de hospitales, Archivo de historias clínicas, Documentación clínica, Historia clínica, Hospital de León, Ponderación de valores económicos.

\section{Title: Evaluation of the medical archive system at the Hospital de León}

Abstract: This paper studies the origin and evolution of three health archives at the Hospital de León that forms part of the Public Health Service of the region of Castilla and León (Spain). The principle of provenance is essential to decide upon the organisation of medical histories archives and all the documents included in them. The study assesses the management of the above mentioned health archives in order to highlight the importance of appropriate cataloguing, shelving and storage of medical records for an efficient day-to-day running of hospitals. The results will be useful in estimating the approximate cost of a proper archives management when taking into account factors such as patients staying longer than expected in hospital or the need to repeat medical tests.

Keywords: Medical archive system, Hospital management, Medical information management, Medical histories, Hospital de León, Health care cost.

Rodríguez López, Ma del Carmen; Díez Díez, Ángela; Gallego Lorenzo, Josefa; Morán Suárez, Marian; Santos de Paz, Lourdes. "Evaluación del sistema archivístico del Hospital de León”. En: El profesional de la información, 2006, mayo-junio, v. 15, $n$. 3, pp. 182-189. 


\section{Introducción}

Corresponden estas líneas a un proyecto de investigación ${ }^{1}$ financiado en la convocatoria de ayudas de la Junta de Castilla y León en materia de gestión sanitaria en el año 2004 (Orden SAN/526/2004, de 7 de abril, $B O C y L$ de 16 de abril de 2004).

Durante los años 1971-1973 emerge un nuevo concepto enunciado como Sistema de Información Sanitaria $(S I S)$, definido como "mecanismos destinados a la recogida, proceso, análisis y transmisión de la información necesaria para la organización y la actividad de los servicios sanitarios, así como para la investigación y la docencia" que incluyen la "información recogida de instituciones que prestan asistencia curativa y preventiva" y "la recuperación de la información científica" ${ }^{2}$. Se constituyen como conjuntos de subsistemas especializados, coordinados entre sí, y no como una unidad orgánica. Este concepto concuerda con la noción coetánea propuesta por Terrada $^{3}$ (1983) de documentación médica entendida como "la aplicación de los saberes y las técnicas de la documentación a los problemas informativos de la medicina, tanto los de la ciencia como los de la práctica médica curativa y preventiva. Se ocupa de la información contenida en cualquier soporte y considera todos sus usos: enseñanza, investigación, práctica clínica y preventiva, planificación, gestión y administración, etc.».

Este planteamiento fue el que llevó a dividir la información médica en dos grandes áreas:

-Médico-científica (knowledge-based medical information).

-Médico-asistencial y sanitaria (Patient-based information ${ }^{4}$ ).

Aunque esta última denominación en inglés parece tener un sentido más restringido que en español, fue el marco propuesto para el proyecto de investigación, porque incluye, además de los datos clínicos individuales, la información de y para la gestión individual de pacientes; para la dirección y administración de los centros asistenciales y sus unidades; y otros sistemas de información sobre las poblaciones enfermas y la

\begin{tabular}{|l|r|c|c|c|}
\hline Año & $\begin{array}{c}\text { No } \\
\text { camas }\end{array}$ & 2002 & 2003 & 2004 \\
\hline No ingresos & 850 & 28.563 & 31.995 & 32.849 \\
\hline $\begin{array}{l}\text { No citas de } \\
\text { consulta }\end{array}$ & 850 & 202.285 & 209.925 & 221.865 \\
\hline $\begin{array}{l}\text { No de } \\
\text { urgencias }\end{array}$ & 850 & 98.942 & 107.508 & 110.408 \\
\hline & 329.790 & 349.428 & 365.122 \\
\hline
\end{tabular}

Tabla 2. Actividades hospitalarias desde el año 2002

\begin{tabular}{|c|c|}
\hline $\begin{array}{l}\mathrm{N}^{0} \text { de } \mathrm{HC} \text { asignadas en el } \\
\text { fichero maestro de pacientes }\end{array}$ & 486.526 \\
\hline $\begin{array}{l}\text { No } \mathrm{HC} \text { asignadas en el año } \\
2004\end{array}$ & 13.526 \\
\hline $\mathrm{N}^{\circ}$ de $\mathrm{HC}$ activas & 119.788 \\
\hline $\mathrm{N}^{\circ}$ de $\mathrm{HC}$ pasivas & 322.053 \\
\hline $\mathrm{N}^{\circ}$ de $\mathrm{HC}$ en histórico & 322.053 \\
\hline $\begin{array}{l}\mathrm{N}^{0} \text { de } \mathrm{HC} \text { integradas en el } \\
\text { archivo central }\end{array}$ & 119.788 \\
\hline $\begin{array}{l}\mathrm{N}^{\circ} \text { de } \mathrm{HC} \text { no devueltas al } \\
\text { archivo (morosos) }\end{array}$ & 7.597 \\
\hline Duplicados & Resto \\
\hline
\end{tabular}

Tabla 1. Estado de la documentación en 2004

evaluación de la asistencia, como los registros específicos de enfermedades.

La asimilación y aplicación de nociones de information science ha permitido superar la organización administrativa clásica de los archivos clínicos y desarrollar un nuevo modelo, el Sistema de Recuperación de la Información Clínica ${ }^{5}$ que significa formular el núcleo conceptual con la aplicación de nociones como documento primario, unidad documental, unidad contable, perfil documental de la historia clínica, indización de la historia clínica, archivos activo e histórico, recuperación selectiva, etc.

\section{«Los conocimientos de archivística, análisis documental y recuperación de la información no se contemplaban en la gestión diaria del hospital»}

Las tecnologías de la información y de las comunicaciones hacen llegar a médicos y resto de profesionales sanitarios la historia clínica (HC), los informes y pruebas médicas complementarias de un determinado paciente en el momento preciso. Esta accesibilidad y disponibilidad de la información requiere, indefectiblemente una mayor normalización en la gestión del archivo. Los conocimientos de archivística, análisis documental y recuperación de información no se contemplan en la gestión diaria del hospital, carencia que resulta palpable y que dificulta enormemente el trabajo. Un sistema pequeño como el que se sometió a estudio ha mostrado las premisas anteriores, evidenciado diferentes usos archivísticos y diferente eficacia en su funcionamiento. A mayor rigor en el análisis documental menor número de duplicados y mayor satisfac- 


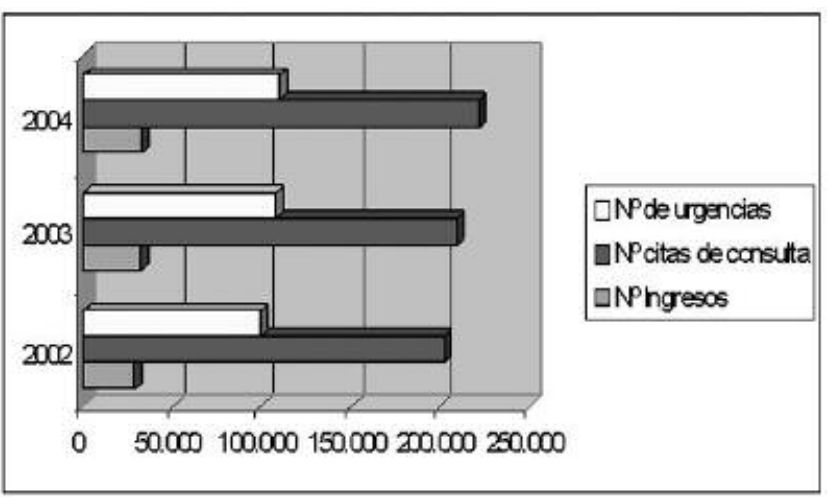

Figura 1. Actividades hospitalarias desde el año 2002

ción entre los usuarios. La divulgación en todos los centros de estos resultados resultaría económicamente rentable para la gestión del sistema hospitalario.

\section{Objetivos.}

-Identificar los órganos productores de la documentación.

— Conocer la estructura orgánica de cada centro.

—Establecer la dependencia jurídica y jerárquica del archivo de HCs.

- Censar el volumen documental.

-Estudiar el tratamiento archivístico de las HCs y los sistemas de recuperación.

-Ponderar el impacto de la gestión de las HCs en los costes generales del Hospital de León mediante el cálculo en el Servicio de Urología, como modelo a exportar al resto de los centros.

\section{Metodología.}

Trabajo en dos secuencias diferentes, la primera destinada al acopio de información y la segunda al proceso de datos:

2.1. Recogida de información, subdividida a su vez en tres fases:

a. Revisión y validación de la legislación y bibliografía acudiendo a repertorios de jurisprudencia, bases de datos, webs y centros de información bibliográfica. Manejo de documentos fundacionales en el archivo de cada centro hospitalario, así como la consulta de otros: Diocesano de León, Catedralicio, Histórico Provincial de León, Municipal de León, Municipal de Astorga, Histórico Nacional y General de Simancas.

b. Trabajo de campo: auditoría parcial de la información centrada en el archivo mediante cuestionarios elaborados ad hoc para:

— Identificar tareas.
- Relacionar las tareas y funciones concretas de cada individuo y la necesidad de disponer de información.

- Reconocimiento de las áreas con mayores requerimientos de información y evaluar el nivel de satisfacción con la proporcionada en la actualidad.

- Establecer las vías utilizadas por los empleados y la Dirección para dar respuesta a estas necesidades.

- Evaluación por los trabajadores del servicio de archivo existente.

c. Entrevistas selectivas a determinados miembros de la organización, asumiendo el incremento de coste, sobre todo en tiempo, y evaluando los beneficios que se derivarán de ellas. La entrevista, basada en preguntas abiertas y semiestructuradas o dirigidas, permite ampliar, matizar o aportar ideas nuevas que el cuestionario, basado principalmente en preguntas cerradas, no permite expresar.

2.2. Procesado de los datos y de la información obtenida.

A partir de los datos conseguidos resultó posible establecer algunas correlaciones hasta determinar: a qué función o funciones responden los diferentes tipos, es decir, lo que relaciona la información, los usuarios y la tipología de demanda a la que dan respuesta, qué atributos tiene cada tipo documental, quiénes son sus productores y consumidores, en qué soporte y formato se genera, cuál es su disponibilidad y accesibilidad, y qué grado de integración o aislamiento tiene con el resto de los sistemas de información corporativos o cuáles son los datos y documentos especialmente críticos para alcanzar los objetivos y la misión corporativa.

\begin{tabular}{|l|c|c|}
\multicolumn{1}{|c}{ Año } & $2003 / 2002$ & $2004 / 2003$ \\
\hline No ingresos & $12,02 \%$ & $2,67 \%$ \\
\hline No citas de consulta & $3,78 \%$ & $5,69 \%$ \\
\hline No de urgencias & $8,66 \%$ & $2,70 \%$ \\
\hline
\end{tabular}

Tabla 3. Incrementos anuales

\section{Tradición hospitalaria en León y provincia: El Camino de Santiago}

Ante la proyección internacional que el desarrollo de las peregrinaciones a Santiago adquirió en los últimos decenios del siglo XI, la monarquía comenzó a tutelar caminos y transeúntes que transitaban en todo el espacio castellano-leonés. No se trataba únicamente de la protección al viator sino de controlar la expansión demográfica y el desarrollo económico que consigo traían estos caminantes ${ }^{6}$. La necesidad de protec- 
ción frente a los muchos peligros que acechaban a los peregrinos, mercaderes y repobladores ocasiona que los monarcas dicten toda una serie de medidas y desarrollen actuaciones que reflejan, en aquel momento, la relación del poder público con las vías de comunicación. Ruiz de la Peña aquilata esta relación que, en su opinión, hace de las rutas "vías públicas o caminos públicos" ámbitos de actuación del poder real. De entre las manifestaciones de la relación que los monarcas medievales mantuvieron con las vías públicas y sus usuarios interesó, en este proyecto, la creación y mantenimiento de una red hospitalaria a favor de los peregrinos, y también de los pobres, enfermos, viudas y huérfanos?

\section{«Puede definirse archivo hospitalario como la unidad operativa encargada de la custodia y gestión de las historias clínicas del hospital, así como de organizar y prestar la documentación clínica»}

En El Camino de Santiago también se construyeron los hospitales de San Antonio y San Marcos, el de las Tiendas (a la entrada de Castilla) y el de Santiago, al que acudían en nutrido número los peregrinos. Antecedente del actual Hospital de León es el antiguo de San Antonio Abad con raíz ya en 1084, cuando Don Pelayo, obispo de la diócesis de León ordenó que se construyera una alberguería en la heredad de Santa María (Iglesia Catedral) ${ }^{8}$. San Antonio Abad es el resultado de la fusión de hospitales y alberguerías nacidos en la Alta Edad Media, y de su adscripción a la jurisdicción episcopal y al cabildo catedralicio.

\section{Identificación del órgano productor de la documentación del archivo}

El complejo que integra el Hospital de León está situado actualmente en los Altos de Nava, s/n, 24008 León. Constituido por tres edificios: el antiguo hospital de San Antonio Abad, reubicado en 1919 y destinado en la actualidad a escuela de enfermería. El Hospital Princesa Sofía nacido en paralelo a la conversión del Hospital de San Antonio en el General de León vio colocada su primera piedra en 1972. En torno a estas fechas también la Seguridad Social hubo de elegir para el Hospital Virgen Blanca idéntico lugar. Las obras realizadas en el complejo hospitalario hicieron de los tres antiguos centros uno solo que ha pasado a denominarse Hospital de León.
Hablamos pues de un complejo hospitalario de 850 camas que, desde 1991, realiza la asistencia sanitaria de la provincia que, según datos del Insalud de 1999, abarca una población de 366.385 habitantes, de los que 172.898 corresponden a zonas urbanas (León capital, La Bañeza y Astorga) y 193.487 a municipios rurales.

\subsection{Historia archivística}

Existe constancia del archivo del hospital desde 1922 en San Antonio Abad, donde se anotaba la información de cada paciente en un libro de registro. La edificación de los hospitales Princesa Sofía y Virgen Blanca motivó la creación de un espacio específico para sus dependencias. Actualmente el archivo ha obtenido una mayor consideración y se encuentra incluido dentro de los servicios del hospital. Antes se localizaba en tres lugares distintos en función de las etapas:

\section{- Activo: en el Hospital Virgen Blanca.}

- Semi-pasivo: en el sótano del Hospital Princesa Sofía. Contiene las HCs que no han experimentado movimientos durante un período mínimo de 7 años.

- Pasivo: etapa inactiva de la documentación e histórica de archivo, localizado en una nave situada en Carvajal de la Legua.

Terminado el traslado al nuevo emplazamiento, el sistema archivístico (la externalización de su gestión se planeó para finales del año 2005) ha quedado establecido de la siguiente manera:

- Activo: alojado en el sótano del hospital y contiene las HCs que se han solicitado en el último año: unas 119.778 .

- Semi-pasivo: ha desaparecido. Una parte de la documentación se ha integrado en el anterior y el resto se ha trasladado al archivo pasivo.

- Pasivo: continúa en la nave situada en Carvajal de la Legua y contiene 322.053 HCs más.

\subsection{Objetivos clave del archivo}

Puede definirse archivo hospitalario como la unidad operativa encargada de la custodia y gestión de las HCs del hospital, así como de organizar y prestar la documentación clínica, tanto para la práctica asistencial como para la docencia, la investigación y el apoyo a la planificación y gestión clínica. En el organigrama del Hospital de León el archivo depende del Servicio de Admisión y Documentación Clínica, con estas funciones:

a. Custodia, organización, recuperación y préstamo de las HCs para asegurar la prestación de la asistencia sanitaria a los pacientes y su continuidad. 
b. Mantenimiento y ordenación de la colección documental abierta, de manera que integre todos los documentos generados por el paciente.

c. Recogida de la documentación que se origina en el centro, conservarla, organizarla y servir los fondos con el principal objetivo de facilitar las funciones a las que está destinada la $\mathrm{HC}$ :

—Asistenciales de los pacientes.

-Científicas, para investigar.

-Docentes, para la formación del personal médico.

—Administrativas y jurídico-legales.

\section{«En el estudio se aprecia la tendencia general de la sanidad a reducir las estancias en los centros hospitalarios, propiciando las recuperaciones en el propio hogar con atención ambulatoria»}

d. Puesto que se trata de documentación de carácter confidencial, es deber del personal del archivo garantizar su protección así como el derecho a la intimidad, conjugándola con el derecho de acceso recogido en el punto anterior.

\subsection{Recursos informativos}

Para cumplir estas funciones el principal recurso es el propio fondo del hospital, compuesto por una única serie documental por cada HC que se le abre a cada paciente con motivo de la asistencia recibida. Los documentos de la HC son los aprobados oficialmente por el mismo, no debiéndose incluir en la historia ningún otro documento a excepción de los procedentes de otros centros. Cada HC está formada, pues, por un conjunto de documentos generados en los distintos episodios que origina el paciente en su paso por el hospital, distribuidos en ocho bloques, a saber:

-Administrativo-legal.

-Informes.

\begin{tabular}{|l|c|c|}
\hline \multicolumn{1}{|c}{ Por día } & Datos anuales \\
\hline TPET & $1.400 /$ día & 511.000 anuales \\
\hline $\begin{array}{l}\text { Porcentaje fallos por } \\
\text { préstamo (PFPre) }\end{array}$ & $10,71 \%$ & \\
\hline $\begin{array}{l}\text { No se localizan en el } \\
\text { archivo }\end{array}$ & & $2 \%$ \\
\hline
\end{tabular}

Tabla 5. Datos de peticiones diarias de HCs y cálculo anual (2004)

\begin{tabular}{|l|l|r|}
\hline \multicolumn{3}{|c|}{ Movimientos de Hc } \\
\hline PPr & Peticiones programadas & $1.300 /$ día \\
\hline PAi & Peticiones aisladas & $100 /$ día \\
\hline FPr & $\begin{array}{l}\text { Porcentaje de fallidas } \\
\text { (motivo) }\end{array}$ & $\begin{array}{r}150 \\
\text { (préstamos) }\end{array}$ \\
\hline EDs & Entradas de dossier al archivo & $1.500 /$ día \\
\hline EPs & Entrada de pruebas sueltas & $50 /$ día \\
\hline
\end{tabular}

Tabla 4. Actividad global del archivo durante el año 2004

—De anamnesis (historial) y de exploración física.

—De evolución.

—Quirúrgico.

—De órdenes médicas.

-De enfermería.

—Exploraciones complementarias.

Las HCs no se destruyen sino que se transfieren al archivo pasivo de Carvajal de la Legua ${ }^{10}$. Este traslado se produce si el paciente ha fallecido, en cuyo caso se las denomina "exitus".

\section{Valor económico de la gestión del archivo en el Servicio de Urología}

El servicio de archivo del Hospital de León gravita en torno al préstamo de HCs, unos 1.300 diarios para consultas programadas, a los que se han de sumar imprevistos. Está dirigido únicamente al personal médico del propio centro, para su uso interno en la institución. El Manual de normas de archivo establece que podrán solicitarse por dos motivos:

\section{a) Asistencial.}

-Urgencias y hospitalización urgente. Previa petición por el facultativo que atiende al paciente.

-Hospitalización programada.

- Consultas externas con cita previa.

-Imprevistos consultas externas. Petición por el facultativo.

- Secretaría para informes.

b) No asistencial.

-Investigación, estudios, estadísticas, etc.: personal facultativo y sanitario no facultativo. Requiere el visto bueno de la Comisión de Investigación.

-Otros motivos (facturación). La Unidad de Cargos a Terceros podrá solicitar en los casos estrictamente necesarios la documentación clínica para la facturación, previa firma del responsable de dicha unidad. 


\begin{tabular}{|c|c|c|c|c|c|}
\hline \multicolumn{7}{|c|}{ Actividad asistencial Servicio de Urología 2004} \\
\hline Camas & Altas & Estancias & Estancia media & Interv. quirúrgicas & Urgencias \\
\hline 18 & 1.067 & 3.242 & 4,75 & 667 & 471 \\
\hline
\end{tabular}

Tabla 6. Actividad asistencial del Servicio de Urología durante el año 2004

Esta casuística, a efectos prácticos, se agrupa en el archivo en cuatro grandes grupos: consultas externas, urgencias, planta (ingresos) y otras unidades peticionarias. Un estudio comparativo de las tres primeras desde 2002 hasta 2004 permite establecer los incrementos habidos en cada una de ellas ${ }^{11}$.

Del estudio de los incrementos se significa el descenso en el número de ingresos frente al aumento de las consultas. Si bien nuestro estudio no entra en cuestiones médicas se aprecia la tendencia general de la sanidad a reducir las estancias en los centros hospitalarios, propiciando las recuperaciones en el propio hogar con atención ambulatoria.

Esta política hospitalaria influye directamente en el índice de ocupación o proporción media anual en que las camas han estado ocupadas respecto a su capacidad máxima. Los datos de ocupación obtenidos para el día 28 de marzo arrojan un resultado del 89,9\%.

\subsection{Actividad global del archivo}

La medición de la actividad se ha llevado a cabo mediante una serie de índices basados en el manual de organización de $\operatorname{archivos}^{12}$, precedido por una serie de cálculos realizados una vez conocida la actividad del archivo durante el año 2004 (tabla 4).

\subsubsection{Cálculos}

El total de peticiones buscadas por día dado (TPet) es igual a la suma de las peticiones programadas y las peticiones aisladas (tabla 5).

$$
\text { TPet }=P P r+P A i
$$

El porcentaje de fallos de localización por préstamo (PFPre):

$$
\text { PFPre }=\frac{F P r}{\text { TPet }} \cdot 100
$$

\subsubsection{Indices}

Evaluación de la capacidad del archivo en cuando a su rentabilidad, eficacia en el proceso y fiabilidad en la situación y ubicación de las HCs.

a. Índice de rentabilidad ${ }^{13}$. Resultado aceptable entre $1 \mathrm{y} 4$.

$$
I_{r}=\frac{511.000}{119.788}=4,2
$$

b. Índice de eficacia ${ }^{14}$.

$$
I_{e}=\frac{1.250}{1.400}=0,89
$$

c. Índice de errores de localización ${ }^{15}$. Valor entre 0 y 0,5 .

$$
I E L=I E L A+I E L P
$$

-Índice de errores de localización de HCs en el $\operatorname{archivo}^{16}$. Se expresa en tanto por uno.

$$
I E L A=\frac{2.396}{119.788}=0,02
$$

-Índice de errores de localización de HCs en préstamo $^{17}$. Se expresa en tanto por uno.

$$
\begin{gathered}
I E L P=\frac{54.750}{119.788}=0,4 \\
I E L=0,02+0,4=0,42
\end{gathered}
$$

\begin{tabular}{|c|c|c|c|c|c|c|c|}
\hline \multicolumn{8}{|c|}{ Estadísticas Servicio de Urología 2004} \\
\hline \multicolumn{5}{|c|}{ Préstamos } & \multicolumn{2}{|c|}{ Peticiones no servidas } & \multirow[t]{2}{*}{ Devoluciones } \\
\hline \multirow[t]{2}{*}{ Destino } & \multicolumn{2}{|c|}{ Asistenciales } & \multicolumn{2}{|c|}{ No asistenciales } & & & \\
\hline & $\begin{array}{c}\text { Consultas } \\
\text { externas }\end{array}$ & $\begin{array}{c}\text { Petición } \\
\text { Inmediata }\end{array}$ & $\begin{array}{c}\text { Estudio e } \\
\text { Invest. }\end{array}$ & Fin administrativo & $\begin{array}{c}\text { No existe } \\
\text { dossier }\end{array}$ & $\begin{array}{c}\text { Dossier } \\
\text { prestado }\end{array}$ & Recuperados \\
\hline $\begin{array}{c}\text { Salida para el } \\
\text { servicio de } \\
\text { Urología }\end{array}$ & 5.685 & 8 & 83 & 1 & 1 & 46 & 5.717 \\
\hline UROC & & 5.693 & 5.777 & 84 & & & \\
\hline
\end{tabular}

El hecho de que los índices de eficacia disminuyan conlleva, implícitamente, que los errores de localización se han elevado considerablemente, ya sea por pérdidas o por errores en la ubicación de las historias instaladas en el archivo. Todo ello acarrea un aumento en los tiempos de búsqueda de las HCs y un mayor número de reclamaciones, que en 2004 llegaron a 161, de

Tabla 7. Estadística de préstamo del Servicio de Urología para consultas (UROC) 


\begin{tabular}{|c|c|c|c|c|}
\hline \multicolumn{5}{|c|}{ Estadísticas Servicio de Urología 2004} \\
\hline \multicolumn{3}{|c|}{ Préstamos por motivo } & \multirow[t]{2}{*}{ Peticiones no servidas } & \multirow[t]{2}{*}{ Devoluciones } \\
\hline Destino & Asistenciales & No asistenciales & & \\
\hline & & Estudio e Invest. & Dossier prestado & Recuperados \\
\hline & & 202 & 4 & 208 \\
\hline UROS & \multicolumn{2}{|c|}{202} & 4 & 208 \\
\hline
\end{tabular}

Tabla 8. Estadística de préstamo del Servicio de Urología para informes (UROS)

las que el $98 \%$ correspondió a HCs prestadas a otros servicios o bien a partes de una historia que habían sido separadas y no resultó posible su localización. El $2 \%$ restante no se detecta en el archivo. En cuanto a los duplicados resulta posible cuantificar los que han sido resueltos en el año (en torno a 1.000) pero no se han podido contabilizar los que se han generado en el mismo período.

\subsection{Actividad del archivo para el Servicio de Urología $(S d U)$}

Se han obtenido los valores de estancia media y de altas en 2004 (tabla 6) del propio Servicio. De los datos se desprende que según el número de camas le corresponde un 2,1\% del total del hospital. El índice de ocupación obtenido para el lunes 28 de marzo de 2005 fue del $100 \%$.

\subsubsection{Cálculos}

De las actividades realizadas por el archivo destacan dos destinos:

—salidas para la consulta de urología, y

- pedidos por la secretaría para informes o para ingresos programados (tablas 7 y 8 ).

Comparando los datos del total del archivo con los obtenidos para el $S d U$, y según el destino de los préstamos, es posible definir el peso de este servicio respecto al total prestado por el hospital, así como los porcentajes dentro del $S d U$ de peticiones no servidas. Cabe resaltar el porcentaje correspondiente a la no

\begin{tabular}{|c|c|}
\hline CmURO & $\begin{array}{c}\text { Coste medio Servicio de } \\
\text { Urología (tomado en un } \\
\text { año) para un diagnóstico } \\
\text { dado }\end{array}$ \\
\hline CmPru & $\begin{array}{c}\text { Coste medio de las } \\
\text { pruebas a repetir }\end{array}$ \\
\hline NEst & $\begin{array}{c}\text { Días de estancia (por } \\
\text { defecto } 1 \text { ) }\end{array}$ \\
\hline Cest & Coste de la estancia $(B O E)$ \\
\hline CS & Coste social \\
\hline CF & Coste final \\
\hline
\end{tabular}

Tabla 10. Interpretación de los factores

\begin{tabular}{|l|c|c|}
\hline Destino & $\begin{array}{c}\text { Préstamos } \\
\text { Servicio de } \\
\text { Urología } \\
\text { respecto a las } \\
\text { HC activas }\end{array}$ & $\begin{array}{c}\text { Peticiones no } \\
\text { servidas, respecto } \\
\text { al total prestado } \\
\text { Servicio de } \\
\text { Urología (motivo) }\end{array}$ \\
\hline UROC & $4,8 \%$ & $\begin{array}{c}0,8 \% \\
\text { (dossier prestado) } \\
0,017 \% \text { (no existe) }\end{array}$ \\
\hline UROS & $0,16 \%$ & $1,9 \%$ \\
(dossier prestado)
\end{tabular}

Tabla 9. Préstamos del Servicio de Urología por destinos

existencia de las HCs, que resulta bastante bajo, respecto al total de HCs del servicio analizado, e inferior al total del hospital (tabla 9).

\section{«Se trató de mostrar que es posible cuantificar el significado económico de los archivos dentro del balance final de la actividad hospitalaria, como ocurre en cualquier empresa»}

\subsection{2. Índices y coste medio de las acciones}

Definido un paciente de mediana edad y sin ningún condicionante especial, tendríamos un índice para medir el coste final de la estancia en el hospital, siendo su valor ideal 1 ó próximo a 1.

$$
\text { Índice }_{1}=\frac{C m U R O}{C F}
$$

Dentro del $S d U$ los costes medios (de los servicios que puede prestar) están relacionados con los grupos de diagnóstico. A ellos se sumarían los gastos, a mayores, causados por la repetición de pruebas relacionadas con la pérdida de documentación producida por un hipotético mal funcionamiento del sistema de archivo. La generación de índices menores de 1 permite definir un incremento en el coste del servicio suministrado al cliente y un mayor tiempo de tratamiento, estancia y recursos empleados. El efecto es directo sobre los dis- 
tintos parámetros de calidad del sistema asistencial. Afecta a la productividad, a la eficiencia, eficacia del centro y por último al usuario (tabla 10).

El coste final producido por este posible mal funcionamiento del servicio de archivo sería ${ }^{18}$ :

$$
C F=C m U R O+N E s t \cdot C E s t+C m P r u+C S
$$

El coste social $C S$ es uno de los factores que está relacionado con el grado de satisfacción del cliente y se contabiliza como el gasto generado a mayores por los retrasos ocasionados por la pérdida de documentación:

$$
C S=\text { Coste_día_trabajo }+ \text { Desplazamiento }
$$

\section{Conclusiones}

Todos los episodios asistenciales generan información que debe incluirse en la HC. Un manejo inadecuado implica necesariamente la repetición de las pruebas respectivas y, en algunos casos el retraso de la intervención. Los costes finales alcanzarían unas cantidades superiores a los gastos previstos inicialmente. El buen funcionamiento de las unidades de archivo es por lo tanto un factor a tener en cuenta para mantener la calidad de todo el sistema. Este proyecto de investigación trató de mostrar que es posible cuantificar el significado económico de los archivos dentro del balance final de la actividad de un hospital como si se tratara de cualquier otro tipo de empresa.

\section{Notas}

1. Dirigido por $\mathbf{M}^{\mathrm{a}}$ del Carmen Rodríguez López como investigadora principal: Evaluación del sistema archivístico sanitario de León. Ponderación del valor económico de su gestión (2004-2005).

2. Háró, A. S. "Information systems for health services at the national level". En: Mclachlan, G. (ed.). Information systems for health services. Copenhagen: World Health Organisation, European Office, 1980, pp. $7-16$.

3. Terrada, M. L. La documentación médica como disciplina. Valencia: Centro de Documentación e Informática Médica, Universidad de Valencia, 1983.

4. Hersh, W. R.; Lunin, L. F. "Introduction and overview". En: Journal of the American Society for Information Science, 1995, n. 46, pp. 726-728.

5. Peris, R. "Archivo central de historias clínicas: integración y recuperación de la información”. En: Medicina española, 1974, n. 72, pp. 35-43.
6. Ruiz de la Peña, J. I. Foncebadón y la asistencia hospitalaria en los puertos de las montañas. Astorga: Centro de estudios astorganos "Marcelo Macías", 2003. Cuadernos, 20. Para todos estos aspectos resulta de obligada referencia la obra publicada por Cavero Domínguez, G.; Martín López, E. Colección documental de la Catedral de Astorga. León: Centro de Estudios "San Isidoro": Caja España de Inversiones: Archivo Histórico Diocesano, 2000. T. II (1126-1299). Col. Fuentes y estudios de historia leonesa, n. 78.

7. Ruiz de la Peña, J. I., Op. cit., p. 9.

8. Lo hace por manda testamentaria otorgada el 13 de diciembre de 1084. Vid. Ruiz Asencio, J. M. Colección documental del Archivo de la Catedral de León (775-1230), IV (1032-1109). León: Centro de Estudios "San Isidoro": Caja España de Inversiones: Archivo Histórico Diocesano, 1990. Doc. núm. 1.236

9. Manual de normas del archivo y uso de la historia clínica. La gestión está centralizada, es decir que toda la documentación generada en cualquiera de los servicios del hospital, tanto Virgen Blanca como Princesa Sofía, se integra en la $\mathrm{HC}$ del paciente.

10. Manual de normas del archivo (...). "Sobre la historia clínica se realizarán procesos de expurgo para facilitar su manejabilidad. Las historias que ocupen más de un sobre podrán dividirse en dos: el activo y el pasivo. El sobre activo contendrá todos los documentos de los últimos episodios del paciente. El sobre pasivo contendrá la documentación de episodios pasados y cerrados, así como las placas radiográficas. Sólo saldrá del archivo este sobre si es solicitado expresamente".

11. Se excluye el año 2005, puesto que no están disponibles aún los datos totales.

12. Sorribas Vivas, M. Organización de archivos clínicos. Barcelona, etc.: Masson, 2001, pp. 94-96.

13. Definido por el número total de HCs buscadas en el año en el depósito, dividido por el número total de HCs archivadas en el archivo activo.

14. Es igual al número de HCs localizadas en el depósito dividido por el número de $\mathrm{HCs}$ buscadas, teniendo en cuenta el número de historias almacenadas tanto en el archivo activo como en el pasivo.

15. Igual a la suma del índice de los errores de localización de HCs en el archivo más el índice de errores de localización de HCs en préstamo.

16. Se calcula dividiendo el número de HCs con errores de localización entre el número total de HCs archivadas.

17. Se calcula dividiendo el número de HCs en préstamo con errores de localización entre el número total de HCs archivadas.

18. En estos costes no se han tenido en cuenta otros gastos generados por bajas.

$M^{a}$ del Carmen Rodríguez López, Ángela Díez Díez, Josefa Gallego Lorenzo, Marian Morán Suárez y Lourdes Santos de Paz, Universidad de León. dphmrl@unileon.es dieadd@unileon.es dphjgl@unileon.es dphmms@unileon.es dphlsp@unileon.es

Ahora renovar (o comenzar) la suscripción a "El profesional de la información" es mucho más ágil y sencillo.

Usted puede gestionar online su suscripción conectándose a esta página web: http://www.elprofesionaldelainformacion.com/suscripciones.htm/

Si lo desea puede comunicar con nosotros dirigiéndose a esta dirección de correo electrónico: suscripciones@elprofesionaldelainformacion.com 